\title{
Metodologia (Methodology)
}

\author{
Análise de sobrevivência \\ aplicada ao estudo da mortalidade de \\ lambari-do-rabo-amarelo \\ Survival analysis \\ applied to mortality study of two \\ spot astyanax \\ Geraldo Magela da Cruz Pereira ${ }^{1}$ \\ Paulo CÉsar Emiliano ${ }^{2}$ \\ Renato Barbosa Ferraz ${ }^{3}$ \\ Ana LÚCia Salaro ${ }^{4}$ \\ \& Raphael Fernandes Soares Alves 5
}

Modelos experimentais são importantes por possibilitarem a obtenção de cenários em que técnicas ou procedimentos podem ser aperfeiçoados e métodos comparados. Esses modelos vêm sendo utilizados amplamente em pesquisas nas áreas médicas e biológicas. Eles auxiliam no entendimento de fenômenos naturais e no desenvolvimento de medicamentos (Ferreira ET AL., 2005; Monteiro ET AL., 2009).

Peixes são utilizados como modelos para estudos biomédicos desde a década de 1970. Esse fato se deve à facilidade de manuseio e ao baixo custo de manutenção. Como exemplo de modelo experimental temos o paulistinha ou zebrafish, que conforme VASCOTTO ET AL., (1997), é um modelo consolidado em várias áreas, sendo utilizado em estudos na neurociência, genômica, genética e para o teste de medicamentos. Liu \& LEACH (2011) ressaltam a importância desse modelo experimental no estudo de câncer em humanos. Pequeno porte, facilidade de manutenção

${ }^{1}$ Doutorando em Estatística Aplicada e Biometria, da Universidade Federal de Viçosa (UFV). Email: geraldomcpereira@gmail.com. ${ }^{2}$ Prof. Adj. Depto Estatistica da UFV. ${ }^{3}$ Doutorando em Ciências Animal da Universidade do Porto (Portugal). ${ }^{4}$ Profa. Tit. do Depto de Biologia Animal, da Universidade Federal de Viçosa (Viçosa, MG) . ${ }^{5}$ Tecnologista de informações geográficas e Estatisticas, Instituto Brasileiro de Geografia e Estatística (IBGE). 
e de reprodução, ciclo de vida curto, e a relativa facilidade apresentada pelo modelo em reproduzir mutações de interesse em estudos na área médica são as principais características desse modelo experimental (SiLVEIRA ET AL., 2012). Recentemente, vem sendo discutida a possibilidade do uso do modelo experimental composto por lambari-do-rabo-amarelo (Astyanax altiparanae).

O lambari-do-rabo-amarelo, também conhecido por tambiú ou piaba, é um peixe de pequeno porte, caracterizado em piscicultura por apresentar hábito alimentar onívoro, alta fecundidade e um ciclo de produção curto, o que garante a existência de animais que atingem o peso comercial em aproximadamente três meses (CotAN ET AL., 2006). Almeida (2007) ressalta que o interesse na espécie é em razão da baixa taxa de mortalidade, do crescimento relativamente rápido, de proles numerosas e da facilidade de manejo tanto em condições de laboratório quanto de campo. Tais características fazem com que o gênero Astyanax seja considerado um bom modelo experimental em estudos que apresentam uma abordagem biológica (Gomes ET AL., 2013; CheHADE ET AL., 2014).

Pesquisas nas áreas de piscicultura ou zoologia frequentemente fazem o uso de porcentagens de sobrevivência para avaliar a mortalidade de animais quando submetidos a condições de interesse diversas. Os métodos de análise de sobrevivência surgem como uma ferramenta extra, que permite analisar de forma detalhada a sobrevivência desses animais, na presença de dados censurados, possibilitando avaliar como a probabilidade de sobrevivência varia com o tempo, e se as correspondentes curvas de sobrevivência diferem estatisticamente entre si.

A análise de sobrevivência constitui-se de um conjunto de métodos estatísticos utilizados para análise de dados, em que a variável de interesse é o tempo decorrido do início do experimento até que um acontecimento ou evento se verifique. Entretanto, nem sempre é possível observar o evento de interesse. Assim, é comum em dados de sobrevivência, a ocorrência de observações censuradas. A censura ocorre quando o indivíduo ou unidade não experimenta o evento de interesse ou experimenta um evento diferente do estudado (KLein \& MoEsChBerger, 2003; Colosimo \& GioLo, 2006).

Embora a análise de sobrevivência tenha se desenvolvido para o estudo de casos nas áreas médica e biológica, a literatura apresenta diversas aplicações desse tipo de análise em outras áreas do conhecimento, tais como sociologia, engenharia, educação, demografia entre outras.

Considerando o exposto, este trabalho tem por objetivo propor o uso de métodos de análise de sobrevivência para analisar o tempo até a 
morte de alevinos (Astyanax altiparanae) em estudos na área de piscicultura.

\section{MATERIALE MÉTODOS}

Métodos estatísticos

A característica importante e que distingue a análise de sobrevivência de outros tipos de análises é a presença de dados censurados, ou seja, a observação parcial ou incompleta da resposta. Isso acontece quando, por alguma razão, o indivíduo ou unidade experimental não experimentou o evento de interesse, sendo assim, há registro parcial de informações, e excluir tais dados da pesquisa acarretaria em uma conclusão equivocada. O que se sabe, na presença de censura, é que o tempo de falha é maior que o tempo de pesquisa ou de censura (Colosimo \& Giolo, 2006).

Em dados de sobrevivência é comum associar a cada indivíduo i, sob estudo, a terna $\left(t_{1}, \delta_{i}, x_{i}\right)$, com $\mathrm{i}=1, \ldots, \mathrm{n}$, em que $t_{\mathrm{i}}$ representa $\mathrm{o}$ tempo de falha ou de censura, $\delta_{\mathrm{i}}$ é uma variável, que indica a ocorrência de uma falha caso $\delta_{\mathrm{i}}=1$, ou uma censura caso $\delta_{\mathrm{i}}=\mathrm{a} \mathrm{e}, \mathrm{x}_{\mathrm{i}}$ representa o valor observado para um dado tratamento.

A distribuição dos tempos de falha pode ser especificada por meio da função de sobrevivência $S(t)$ que é definida como a probabilidade de um indivíduo sobreviver a um certo tempo t. HosMer \& LEMESHOw (2008) a define como a probabilidade de que T exceda um valor $\mathrm{t}$, em um dado intervalo. Em termos probabilísticos segue:

$$
\mathrm{S}(\mathrm{t})=\mathrm{P}(\mathrm{T}>\mathrm{t})=1-\mathrm{P}(\mathrm{T} \leq \mathrm{t}) .
$$

Definida a função de sobrevivência, torna-se importante obter estimativas de sobrevivência para as unidades experimentais. ColletT (2003) cita três classes de modelos para estimação da função de sobrevivência, que podem ser: não paramétricos, paramétricos ou semiparamétricos. Neste trabalho foi utilizado o método não paramétrico, que se caracteriza por não fazer restrição quanto ao tipo de distribuição seguida por $\mathrm{T}$ e tem como limitação o fato de não permitir testar o efeito de muitas covariáveis simultaneamente. No caso não paramétrico podemos citar o estimador de Kaplan-Meier, que teve suas propriedades estatísticas estudadas por KaPlan \& MEIER (1958).

Segundo Collett (2003) e Colosimo \& Giolo (2006), o estimador Kaplan-Meier, também chamado de estimador limite-produto, denotado por $\widehat{\mathrm{S}}(\mathrm{t})$, é o método mais conhecido e utilizado para se obter estimativas para a função de sobrevivência, na presença de uma amostra censurada. Considere uma amostra de $n$ indivíduos de um estudo, em que ocorrem $\mathrm{k} \leq \mathrm{n}$ falhas distintas nos tempos $\mathrm{t}_{1}<\mathrm{t}_{2}<\cdots<\mathrm{t}_{\mathrm{k}}$. 
Segundo Collett (2003) e Colosimo \& Giolo (2006), o estimador de Kaplan-Meier é definido de forma geral por:

$$
\hat{\mathrm{S}}(\mathrm{t})=\prod_{\mathrm{j}: \mathrm{t}_{\mathrm{j}}<\mathrm{t}}\left(\frac{\mathrm{n}_{\mathrm{j}}-\mathrm{d}_{\mathrm{j}}}{\mathrm{n}_{\mathrm{j}}}\right)=\prod_{\mathrm{j}: \mathrm{t}_{\mathrm{j}}<\mathrm{t}}\left(1-\frac{\mathrm{d}_{\mathrm{j}}}{\mathrm{n}_{\mathrm{j}}}\right),
$$

em que $n_{i}$ é o número de indivíduos sob risco em $t_{i}$, ou seja eles não falharam e não foram censurados antes do tempo $t_{j}, j=1,2, \ldots, k$ e $n_{j}$ é número de indivíduos sob risco em, ou seja, eles não falharam e não foram censurados antes do tempo .

A comparação de tratamentos é o objetivo principal na maioria dos estudos na área médica, tal como ocorre em ensaios clínicos. O interesse é avaliar o efeito de um ou mais tratamentos na sobrevivência de um grupo de indivíduos com base em um grupo controle. Desse modo, considerandose o caso em que há dois tratamentos objetiva-se testar as seguintes hipóteses:

$$
H_{0}: S_{1}(t)=S_{2}(t) \text { vs } H_{1}: S_{1}(t) \neq S_{2}(t) \text {. }
$$

Apresentado por MANTEL (1966), o teste logrank é um dos mais utilizados para comparar curvas de sobrevivências de dois ou mais grupos. Segundo LiN \& XU (2009), esse teste é indicado se o pressuposto básico de proporcionalidade das taxas de falha das populações for satisfeito.

O teste logrank fundamenta-se nas medidas UL e VL, que representam, respectivamente, o desvio entre o valor observado e o esperado do número de falhas em um certo tempo t, e VL a variância de UL. Considerando que os tempos de falha são independentes, tem-se:

$$
\mathrm{U}_{\mathrm{L}}=\sum_{\mathrm{j}=1}^{\mathrm{k}}\left(\mathrm{d}_{1 \mathrm{j}}-\mathrm{e}_{1 \mathrm{j}}\right) \text { e } \operatorname{Var}\left[\mathrm{U}_{\mathrm{L}}\right]=\sum_{\mathrm{j}=1}^{\mathrm{k}} \mathrm{v}_{1 \mathrm{j}}=\mathrm{V}_{\mathrm{L}} \text {. }
$$

sendo: $\mathrm{k}$ o índice associado ao maior dos tempos de falha, $\mathrm{d}_{1 \mathrm{j}}$ o número observado de falhas no grupo $1 \mathrm{em}_{\mathrm{j}}, \mathrm{e}_{1 \mathrm{j}} \mathrm{o}$ número esperado de falhas e $\mathrm{v}_{1 \mathrm{j}}$ a variância.

Supondo a não rejeição da hipótese nula então há igualdade entre as curvas de sobrevivência dos dois grupos. Um caminho para averiguar esse fato é comparar o número de indivíduos que falharam nos dois grupos em cada um dos tempos de falha, com o número esperado de falhas sob a hipótese nula.

A estatística do teste é dada por:

$$
\mathrm{W}_{\mathrm{L}}=\frac{\mathrm{U}_{\mathrm{L}}^{2}}{\mathrm{~V}_{\mathrm{L}}} \sim \chi_{1}^{2}
$$


que sobre a hipótese nula segue uma distribuição qui-quadrado com um grau de liberdade.

Segundo Colosimo\& Giolo (2006), o teste logrank pode ser generalizado para mais de duas funções de sobrevivência $r>2$, neste caso, WL segue uma distribuição qui-quadrado com $r-1$ graus de liberdade.

\section{DESCRIÇÃO DOS DADOS}

Os dados utilizados na presente pesquisa são provenientes do estudo realizado no Laboratório de Nutrição de Peixes II do Setor de Piscicultura do Departamento de Biologia Animal da Universidade Federal de Viçosa, em Viçosa, Minas Gerais. Esse estudo é aprovado pela Comissão de Ética no Uso de Animais de Produção (CEUAP/UFV), processo no $21 /$ 2013. Em tal pesquisa, utilizou-se delineamento inteiramente casualizado com cinco dietas (a base de óleos de soja, coco, peixe, linhaça e palma) e cinco repetições. As dietas práticas eram isoproteicas $(32 \% \mathrm{~PB})$ e isoenergéticas (4300 kcal de EB/ $\mathrm{kg}$ de ração) diferindo apenas na fonte de lipídio (Tabela 1). Peixes em crescimento $(1,44 \pm 0,20 \mathrm{~g} \mathrm{e} 3,23 \pm 0,20 \mathrm{~cm})$ foram distribuídos em 25 aquários, na densidade de 20 peixes por aquário e cada aquário foi considerado como uma unidade experimental.

Os aquários foram mantidos em sistema de recirculação de água com vazão de 1 litro/min, dotados de sistema de filtragem mecânica, biológica, aeração contínua e temperatura $\left(27,17 \pm 0,92^{\circ} \mathrm{C}\right)$ controlada por um aquecedor e termostato central. $\mathrm{O} \mathrm{pH}$ foi mantido em 7,6 $\pm 0,357$ e a amônia em $0,2 \pm 0,11 \mathrm{mg} / \mathrm{L}$, e oxigênio dissolvido entre $6,5 \mathrm{a} 7,5 \mathrm{mg} / \mathrm{L}$. A alimentação dos peixes foi feita diariamente, quatro vezes por dia, manualmente, até a aparente saciedade dos peixes.

Para o estudo da sobrevivência dos peixes, cada indivíduo foi considerado como uma unidade experimental, assim, para cada um dos tratamentos havia 100 peixes em análise, totalizando 500 peixes no estudo. Nesse caso, a variável de interesse foi o tempo até a morte de um peixe. O experimento foi conduzido minuciosamente, de modo que cada aquário garantisse semelhante condição de sobrevivência aos peixes.

MÉTOdOS DE ANÁLISE

Para possibilitar a análise estatística dos dados de sobrevivência, o evento de interesse para os dados reais foi definido como a morte de um peixe. Portanto, o tempo de falha é o tempo decorrido do início do estudo até a morte do peixe. Peixes que ao final do estudo não experimentaram o evento de interesse foram censurados, sendo assim, ocorreu censura do Tipo I. O estudo teve início no dia 20/10/2014 e término no dia 03/02/ 2015, assim, o tempo de censura foi de 107 dias. A cada unidade 
Tabela 1. Composição percentual e química das dietas experimentais utilizadas na alimentação do lamberi-do-rabo-amerelo (Astyanax altiparanae) em fase de crescimento.

\begin{tabular}{|c|c|c|c|c|c|}
\hline \multicolumn{6}{|c|}{ Fontes de lipídios } \\
\hline Ingredientes & Peixe & Soja & Linhaça & Palma & Coco \\
\hline Farelo Soja & 53,50 & 53.50 & 53,50 & 53,50 & 53,50 \\
\hline Glúten de Milho & 7,00 & 7,00 & 7,0 & 7,00 & 7,00 \\
\hline Fubá Milho & 14,05 & 14,05 & 14,05 & 14,05 & 14,05 \\
\hline Farelo Trigo & 14,50 & 14,50 & 14,50 & 14,50 & 14,50 \\
\hline L - Lisina & 0,33 & 0,33 & & 0,33 & 0,33 \\
\hline DL - Metionina & 0,30 & & & 0,30 & 0,30 \\
\hline FosfBicálcico & 3,80 & 3,80 & & 3,80 & 3,80 \\
\hline Sal comum & 0,25 & 0,25 & & 0,25 & 0,25 \\
\hline Premix vit $^{1}$. & 0,15 & 0,15 & & 0,15 & 0,15 \\
\hline Premix min ${ }^{2}$. & 0,10 & & & 0,10 & 0,10 \\
\hline & 0,02 & & & 0,02 & 0,02 \\
\hline Óleo Peixe & 6,00 & 0,00 & & 0,00 & 0,00 \\
\hline Óleo & 0,00 & 6,00 & & 0,00 & 0,00 \\
\hline inhaca & 0,00 & 0,00 & & 0,00 & 0,00 \\
\hline & 0,00 & 0,00 & & 0,00 & 6,00 \\
\hline & 0,00 & 0,00 & 0,00 & 6,00 & 0,00 \\
\hline \multicolumn{6}{|c|}{ Composição química* } \\
\hline Prot Bruta & 35,46 & 33,27 & 35,48 & 35,58 & 35,57 \\
\hline Lipídios Totais & 8,48 & 9,32 & 8,58 & 8,95 & 8,61 \\
\hline Cinza & 8,02 & 7,96 & 8,05 & 7,89 & 7,91 \\
\hline \multicolumn{6}{|c|}{ 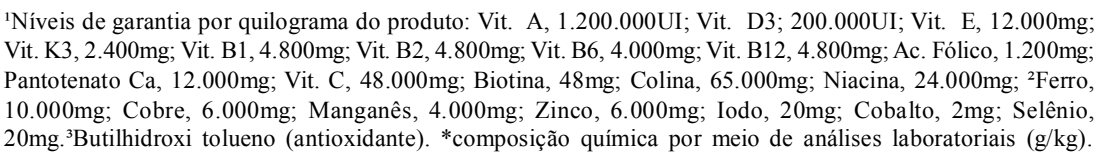 } \\
\hline \multicolumn{6}{|c|}{$\begin{array}{l}\text { experimental foi associado um tempo de falha e uma variável indicadora } \\
\text { de censura } \delta \text {. Diariamente, os aquários eram checados e registrava-se, } \\
\text { caso ocorresse, o dia do evento de interesse. } \\
\text { Para avaliar a mortalidade dos peixes em cada um dos tratamentos, } \\
\text { foram estimadas as funções de sobrevivência pelo método Kaplan-Meier, } \\
\text { em seguida, foram construídos gráficos para as curvas de sobrevivência. } \\
\text { Finalmente, as funções de sobrevivência foram comparadas por meio do } \\
\text { teste logrank, com o intuito de observar se havia diferença significativa } \\
\text { entre os tratamentos. } \\
\text { As comparações múltiplas das funções de sobrevivência foram } \\
\text { realizadas levando em conta a correção de Bonferroni }(\alpha / \text { c), em que } \alpha \\
\text { é o nível de significância ( } 5 \% \text { e c o número de comparações feitas. } \\
\text { Considerando o grande número de comparações }(10) \text {, a correção foi } \\
\text { adotada por permitir uma diminuição do erro tipo I. }\end{array}$} \\
\hline
\end{tabular}


A análise dos dados foi realizada por meio do software $\mathrm{R}$, cujas rotinas foram obtidas por adaptações daquelas encontradas em CoLosimo \& Giolo (2006).

\section{RESULTADOS E DISCUSSÃO}

Do total de peixes envolvidos no estudo, somente 134 morreram, experimentando o evento de interesse. A porcentagem total de falha foi de 26,8\%. Nota-se uma elevada taxa de censura nos dados (Tabela 2). A porcentagem de censura foi maior nas dietas à base de Soja, Peixe e Linhaça. Observa-se também que os menores tempos de falha e as menores taxas de censura foram encontradas nas dietas à base de Coco e Palma. Nota-se que o máximo dos tempos de falha foi de 107 dias para todos os tratamentos. Isso se deve à ocorrência de empate entre os tempos de falha e de censura, segundo Colosimo \& Giolo (2006), se $\mathrm{T} \leq \mathrm{C}$, então $\delta=1$, o que determina a ocorrência de uma falha. $\mathrm{O}$ tempo médio de sobrevivência $\left(\mathrm{t}_{\mathrm{m}}\right)$, foi obtido pelo cálculo da área sob a curva de sobrevivência para cada um dos tratamentos, conforme sugerido por Colosimo \& Giolo (2006).

Tabela 2. Dados descritivos para cada uma das cinco dietas com diferentes fontes de lipídios

\begin{tabular}{lcccc}
\hline & Tratamento & Mínimo & Máximo & Censura (\%) \\
\hline Soja & 73 & 105,90 & 107 & 94 \\
Coco & 44 & 96,57 & 107 & 49 \\
Peixe & 59 & 104,53 & 107 & 86 \\
Linhaça & 81 & 106,02 & 107 & 91 \\
Palma & 52 & 97,14 & 107 & 46 \\
\hline
\end{tabular}

$\mathrm{t}_{\mathrm{m}}$ é o tempo médio de vida.

Tabela 2 dados descritivos para cada uma das cinco dietas com diferentes fontes de lipídiosPara cada uma das dietas, foram estimadas as curvas de sobrevivência via Kaplan-Meier (Fig. 1). Além da análise gráfica, foi utilizado, também, o teste logrank para verificar se tais diferenças são estatisticamente significativas. Percebe-se pela figura, que as curvas de sobrevivência começam a divergir para valores de tempo superiores a 50 dias. Observa-se que a probabilidade de sobrevivência para peixes com dietas à base de óleo de Coco e Palma decrescem drasticamente para valores de tempo superiores a 70 dias, enquanto a probabilidade de sobrevivência de peixes com dietas à base de óleo de Soja, Peixe e Linhaça decresce pouco com o tempo, ou seja, 


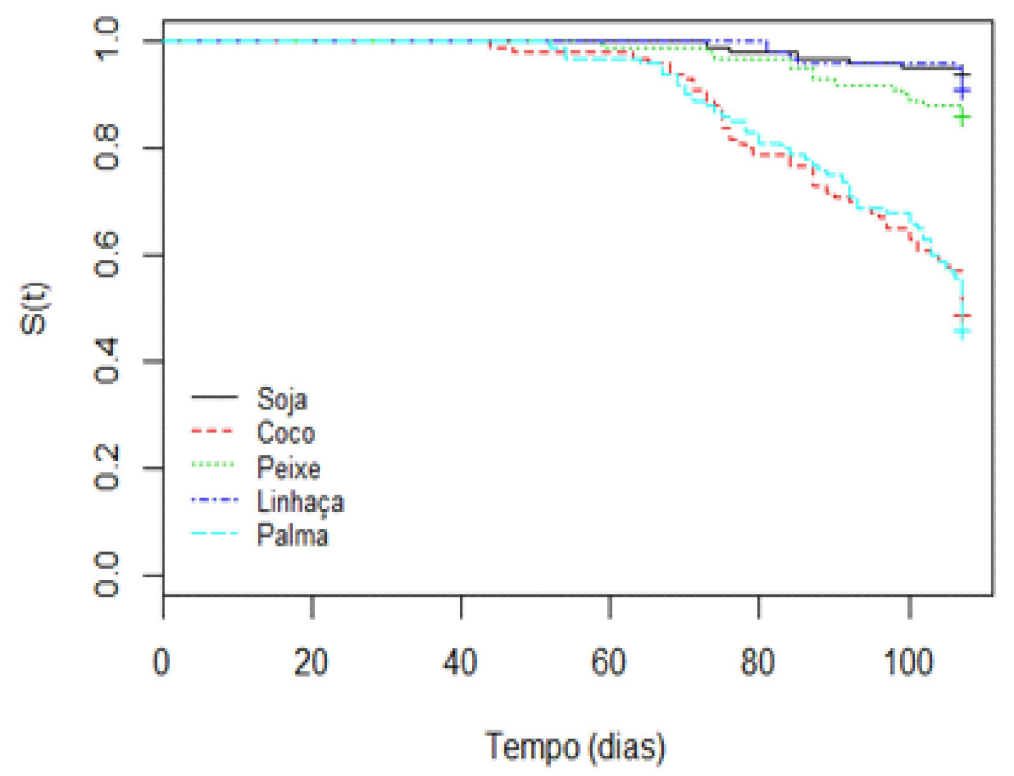

Fig. 1. Estimativas de sobrevivência, pelo método Kaplan-Meier para cada um dos tratamentos.

observa-se que os peixes que receberam esses tratamentos tiveram maiores chances de sobrevivência.

Percebe-se a existência de dois grupos de curvas de sobrevivência (Fig. 1), o primeiro composto pelas dietas à base de Soja, Linhaça e Peixe, e o segundo grupo composto pelas dietas à base de Palma e Coco. Uma interpretação subjetiva nos leva a concluir que, dentro dos grupos, as curvas de sobrevivência são semelhantes, porém, quando comparados os grupos, as curvas aparentam ser diferentes, e isso será comprovado pelo teste logrank.

Sob a hipótese nula de igualdade entre as curvas de sobrevivência, foi aplicado o teste logrank considerando inicialmente as cinco curvas de sobrevivência conjuntamente, o que forneceu o valor $=123 \mathrm{com} 4$ graus de liberdade para a estatística do teste logrank e o correspondente valor p inferior a 0,05 . Desse modo, rejeita-se a hipótese nula e, portanto, pode-se afirmar que existe diferença significativa entre pelo menos duas curvas de sobrevivência $(\mathrm{p}<0,05)$.

Verificada a existência de diferença entre os tratamentos, o passo seguinte constituiu-se em identificar quais curvas eram estatisticamente 
diferentes, comparando-as aos pares pelo teste logrank (Tabela 3). Fazendo uso da correção de Bonferroni, sugerida por Colosimo \& Giolo (2006), foi adotado o nível de significância de $0,05 / 10=0,005$, sendo 10 o número total de comparações dois a dois entre os cinco tratamentos (dietas).

Tabela 3. Valores $p$ referentes ao teste não paramétrico logrank com a correção Bonferroni.

\begin{tabular}{ccccc}
\hline & Soja & Coco & Peixe & Linhaça \\
\hline Coco & $<1,37 \times 10^{-12 *}$ & & & \\
Peixe & $0,061^{n s}$ & $<1,91 \times 10^{-8 *}$ & & \\
Linhaça & $0,439^{n s}$ & $<2,68 \times 10^{-11 *}$ & $0,25^{n s}$ & \\
Palma & $<1,16 \times 10^{-13 *}$ & $0,803^{n s}$ & $<2,94 \times 10^{-9 *}$ & $<2,3 \times 10^{-12 *}$ \\
\hline
\end{tabular}

Considerando o nível de significância de $5 \%$ e a correção de Bonferroni, percebe-se que existem diferenças significativas entre os dois grupos mencionados anteriormente. Concordando com a interpretação gráfica, dentro dos grupos, não existem diferenças significativas entre as curvas de sobrevivência. Assim, as curvas de sobrevivência correspondentes às dietas à base de óleo de Soja, Peixe e Linhaça são estatisticamente iguais, podendo-se dizer a mesma coisa acerca das curvas das dietas à base de óleo de Coco e Palma. Por fim, tem-se, também, que as demais combinações das curvas duas a duas diferem-se estatisticamente.

\section{CONCLUSÕES}

O método Kaplan-Meier, aliado ao teste logrank, permitiu comparar de modo satisfatório as curvas de sobrevivência dos peixes em cada uma das dietas. Pelos métodos estatísticos utilizados, percebe-se que a análise de sobrevivência possibilita um estudo completo e detalhado de dados de sobrevivência na área de piscicultura. As curvas de sobrevivência permitem observar os diferentes caminhos seguidos pela sobrevivência dos peixes nos diferentes tratamentos, o que não é possível ao se avaliar as porcentagens de sobrevivência em cada tratamento.

\section{SUMÁRIO}

O objetivo deste trabalho foi aplicar métodos de análise de sobrevivência para avaliar a mortalidade de peixes, quando submetidos a cinco dietas com diferentes fontes de lipídios (óleos de soja, coco, peixe, linhaça e palma). Os tempos de falha foram obtidos experimentalmente 
ao submeter alevinos a cinco dietas, sendo cada uma aplicada a 100 peixes. Aqueles que ao final do estudo não falharam (morreram) foram considerados como observações censuradas, caracterizando a ocorrência de censura do Tipo I. A análise da mortalidade dos peixes foi feita estratificando os tempos em cada um dos cinco tratamentos. Assim, para cada tratamento, foram obtidas estimativas de sobrevivência pelo método Kaplan-Meier, em seguida, as curvas de sobrevivência nos respectivos tratamentos foram comparadas, via teste logrank, o que permitiu observar a existência de diferenças significativas entre os tratamentos. Concluise que os métodos da análise de sobrevivência permitem avaliar de modo satisfatório a mortalidade dos peixes, possibilitando observar o comportamento da curva de sobrevivência desses animais em cada um dos tratamentos em função do tempo.

PalaVRas-ChaVe: não-paramétricos; Kaplan-Meier; logrank; Astyanax altiparanae

\section{SUMMARY}

The objective of this study was to apply survival analysis methods to evaluate fish mortality when exposed to five diets composed of different sources of lipid (soy, coconut, fish, linseed and palm oils). Failure time was experimentally obtained when exposing alevins to five diets and each of them were applied to 100 fish. The ones which did not failure (died) at the end of the study were considered as censored observations characterizing the occurrence of type I censoring. Fish mortality was analyzed stratifying the times in each of the five treatments. Thus, for each treatment, survival estimations were obtained through Kaplan-Meier method, subsequently, survival curves in the respective treatments were compared via logrank test which permitted to observe the existence of meaningful differences between the treatments. On balance, the utilization of survival analysis method permits to evaluate satisfactorily fish mortality enabling to observe the survival curve behavior of the fish in each treatment as a function of the time.

KeYwORDS: nonparametric; Kaplan-Meier; logrank test, Astyanax altiparanae

Agradecimentos - À Fundação de Amparo à Pesquisa de Minas Gerais (FAPEMIG) e ao Conselho Nacional de Desenvolvimento Científico e Tecnológico (CNPq), pelas bolsas concedidas de mestrado e Ic. Essas bolsas de mestrado e Ic, devem ser agradecidas também ao Ministry of Science and Technology (MCT) and the Ministry of Fisheries and Aquaculture (MPA), for the award of the scholarship of the announcement Public Call MCT /CNPq/CTAgronegócio/ MPA N 25/2010 — Human Resources Training in Fisheries and Aquaculture. 


\section{BIBLIOGRAFIA}

AlmeidA, R. B. C. 2007. Astyanax altiparanae (Pisces, Characiformes) como modelo biológico de espécie de peixe para exploração zootécnica e biomanipulação. Tese (Doutorado em ciências biológicas - Zoologia). Universidade Estadual Paulista Júlio de Mesquita Filho, Botucatu, SP.

Chehade, C.; M. Cassel; M. I. Borella \& F. G. Costa. 2014. Morphologic study of the liver of lambari (Astyanax altiparanae) with emphasis on the distribution of cytokeratin. Fish Physiol Biochem, 40(2): 571576.

Collet, D. 2003. Modelling Suvival Data in Medical Research. 2. ed. New York: Chapman \& Hall: 391 pp.

Colosimo, E. A. \& S. R. Giolo. 2006. Análise de Sobrevivência Aplicada. 1. ed. São Paulo: Edgard Blucher: 370 pp.

Cotan, J. L. V.; E. A. T. Lanna; M. A. D. Bomfim; J. L. Donzele; F. B. RibeIro \& M. A. SERAFINI. 2006. Níveis de energia digestível e proteína bruta em rações para alevinos de lambari tambiú. Revista Brasileira de Zootecnia, 35(3): 634-640.

Ferreira, L. M.; B. Hochman \& M. V. J. Barbosa. 2005. Modelos experimentais em pesquisa. Acta Cirúrgica Brasileira, 20(2): 2834.

Gomes, C. C.; F. G. Costa \& M. I. Borella. 2013. Distribution of GnRH in the brain of the freshwater teleost Astyanax altiparanae. Micron, 52(53): 33-38.

Hosmer, D. W. \& S. Lemeshow. Applied survival analysis. 2008. 2. ed. New Jersey: John Wiley and Sons: 392 pp.

Kaplan, E. L. \& P. Meier. 1958. Nonparametric estimation from incomplete observations. Journal of the American Statistical Association, 53(282): 457-481.

Klein, J. P. \& M. L. MoeschBerger. 2003. Survival analysis techniques for censored and truncated data. 2. ed. New York: Springer: 536 pp.

LIN, X. \& Q. XU. 2009. A new method for the comparison of survival distributions. Pharmaceutical Statistics, 9: 67-76.

Liu, S. \& S. D. LeACH. 2011. Zebrafish Models for Cancer. The Annual Review of Pathology: Mechanisms of Disease, 6: 71-93. 
Mantel, N. 1966. Evaluation of survival data and two new rank order statistics arising in its consideration. Cancer Chemotherapy Reports, 50(3): 163-170.

Monteiro, R.; R. Brandau; W. J. Gomes \& D. M. Braile. 2009. Tendências em experimentação animal. Revista Brasileira de Cirurgia Cardiovascular, 24(4): 506-513.

R Development Core Team. R: 2017. A language and environment for statistical computing. Vienna, Austria: R Fundation for Statistical Computing. Disponível em: $<$ http://r-project.org $>$.

Silveira, T. R.; A. C. Schneider \& T. O. Hammes. 2012. Zebrafish: modelo consagrado para estudos de doenças humanas. Ciência e Cultura, 64(2): 4-5.

Vascotto, S. G.; Y. Beckham \& G. M. Kelly. 1997. The zebrafish swin to fame as an experimental model in biology. Biochem. Cell. Biol., 75: 479-485.

Recebido 3de março de 2017. 